The bidirectional relationship between exercise and sleep:
Implications for exercise adherence and sleep improvement
Am J Lifestyle Med. $2014 ; 8(6): 375-379$. doi:10.1177/1559827614544437.
Christopher E. Kline, Ph.D.
Department of Psychiatry, University of Pittsburgh, Pittsburgh, PA
Abstract
Exercise has long been associated with better sleep, and evidence is accumulating on the efficacy
of exercise as a nonpharmacologic treatment option for disturbed sleep. Recent research, however,
has noted that poor sleep may contribute to low physical activity levels, emphasizing a robust
bidirectional relationship between exercise and sleep. This article will briefly review the evidence
supporting the use of exercise as a nonpharmacologic treatment for sleep disturbance, outline
future research that is needed to establish the viability of exercise as a behavioral sleep treatment,
describe recent research that has emphasized the potential influence of poor sleep on daytime
activity levels, and discuss whether improving sleep may facilitate adoption and/or better
adherence to a physically active lifestyle. With poor sleep and physical inactivity each recognized
as key public health priorities, additional research into the bidirectional relationship between
exercise and sleep has significant implications for facilitating greater exercise adherence and
improving sleep in society.

\title{
Keywords
}

exercise; insomnia; obstructive sleep apnea; physical activity; sleep

Sufficient sleep is essential for optimum health—just a few of the numerous processes occurring during sleep include memory consolidation, clearance of brain metabolites, and restoration of nervous, immune, skeletal, and muscular systems. ${ }^{1}$ Virtually all bodily systems are impacted by poor or inadequate sleep, and chronic sleep disturbance predisposes an individual to cardiovascular disease, metabolic dysfunction, psychiatric disorders, and early mortality. ${ }^{1,2}$

Despite the significance of adequate sleep, insufficient or disturbed sleep is extremely common. For instance, thirty percent of employed adults report obtaining 6 or fewer hours of sleep per night, ${ }^{3}$ approximately one-third of all adults report significant sleep complaints, ${ }^{4}$ and the two most common sleep disorders, insomnia and sleep-disordered breathing (SDB), each have prevalence rates exceeding $10 \%$ in the adult population. ${ }^{5,6}$ Unfortunately, current approaches to treatment are limited. For poor sleep quality and insomnia complaints, prescription hypnotic medications offer short-term efficacy but are

Corresponding Author Information: Christopher E. Kline, Ph.D., Department of Psychiatry, University of Pittsburgh, 3811 O'Hara Street, Room E-1124, Pittsburgh, PA 15213, Tel: 412-586-9434, Fax: 412-246-5300, klinec@upmc.edu. 
plagued by concerns about dependency, hazardous side effects, and long-term health risk. ${ }^{7}$ In contrast, cognitive-behavioral therapy for insomnia (CBT-I) provides greater long-term efficacy and fewer side effects than hypnotics; however, availability remains restricted. ${ }^{8}$ Meanwhile, the primary treatment option for SDB, continuous positive airway pressure (CPAP), significantly reduces SDB symptoms when used, but compliance remains problematic. $^{9}$

Due to its wide-ranging health benefits, minimal cost and side effects, and accessibility, exercise is an attractive nonpharmacologic treatment option for disturbed sleep. ${ }^{10}$ Recent research, though, has shown that poor sleep may hamper efforts to be physically active, emphasizing the bidirectional relationship between exercise and sleep. This article will briefly summarize the current evidence supporting the use of exercise in the management of sleep problems, suggest future research that would help establish the viability of exercise as a behavioral sleep medicine treatment option, review recent research showing that sleep and sleep timing may contribute to physical inactivity, and discuss whether improving sleep may facilitate a physically active lifestyle.

\section{Exercise as a Treatment for Disturbed Sleep}

Exercise has long been associated with better sleep. ${ }^{11}$ Despite surprisingly little experimental research involving patients with significant sleep disturbance or sleep disorders, the available evidence suggests that exercise holds promise as a nonpharmacologic therapy for adults with poor or disordered sleep. ${ }^{10}$

Most studies that have examined the effects of exercise training on general poor sleep quality (i.e., subclinical sleep complaints) have focused on older adults, among whom sleep complaints are extremely prevalent. A recent meta-analysis of six studies found that exercise training resulted in modest improvements in subjective sleep quality in middle- to olderaged adults with sleep problems. ${ }^{12}$ However, other trials have found minimal to no improvements in sleep following exercise training (e.g., ${ }^{13}$ ) and objective sleep parameters have rarely been found to change in the few trials that have utilized actigraphy or polysomnography. ${ }^{14,15}$ Although there are only three published studies to date, research that has focused on adults diagnosed with chronic insomnia have provided much more consistent —and promising — results on the effect of exercise on sleep. ${ }^{16-18}$ As a prominent example, Reid and colleagues found that 4 months of aerobic exercise training in a sample of older adults with insomnia significantly improved sleep quality while also reducing daytime sleepiness and depressive symptoms. ${ }^{17}$ Thus, exercise may hold the most promise for those with more severe or more chronic sleep disturbance.

Exercise training also reduces SDB severity, with obstructive sleep apnea (OSA) the most common type of SDB examined. A recent meta-analysis of five studies found that exercise training reduced OSA severity by $32 \%$ despite a nonsignificant decrease in body mass index(BMI). ${ }^{19}$ In the largest randomized trial to date, Kline and colleagues found that 12 weeks of moderate-intensity aerobic and resistance exercise resulted in a $25 \%$ reduction in OSA severity despite less than $1 \mathrm{~kg}$ of weight loss. ${ }^{20}$ Notably, exercise training also led to 
better subjective and objective sleep and improvements in daytime functioning (e.g., quality of life, depressive symptoms, vigor, fatigue) in this sample. ${ }^{21}$

It is less understood whether a single exercise bout impacts the corresponding night's sleep in adults with poor sleep. Although a previous meta-analysis reported modest improvements in sleep following an acute bout of exercise, these results were based on studies that only included good sleepers. ${ }^{22}$ In contrast, only two studies have examined this question in poor sleepers, both involving adults diagnosed with chronic insomnia. Passos and colleagues found that an acute bout of moderate-intensity aerobic exercise, but not high-intensity aerobic exercise or high-intensity resistance exercise, improved sleep compared to a baseline night in a sample of middle-aged adults. ${ }^{23}$ In contrast, Baron and colleagues found no relationship between single sessions of moderate-intensity aerobic exercise and sleep during the corresponding night across a 4-month exercise intervention for 11 older adults. ${ }^{24}$ The exercise regimen did, however, result in significant improvements in sleep efficiency and sleep duration at post-intervention compared to baseline. ${ }^{24}$ Overall, then, the divergent findings from these two studies suggest that the sleep-enhancing effects of exercise may not manifest initially.

\section{Exercise to Improve Sleep: Future Research Needs}

Overall, the available evidence certainly suggests that exercise could be a valuable behavioral therapy for poor or disordered sleep. However, many unanswered questions need resolved before exercise can be optimally prescribed as a behavioral treatment option for disturbed sleep. Perhaps the most pressing need is for larger-scale experimental studies to be conducted as, for both insomnia/poor sleep quality and SDB, the current evidence base is too small to provide well-informed recommendations. For instance, recent meta-analyses summarizing the effect of exercise training on subjective sleep quality and OSA severity included only 305 (6 trials) and 129 participants (5 trials), respectively. ${ }^{12,19}$ Larger sample sizes will also allow researchers to examine whether specific participant characteristics can predict the efficacy of exercise on sleep outcomes; while most studies have found that exercise, on average, modestly improves sleep parameters and OSA severity, it is plausible that individuals will respond differently depending upon specific attributes (e.g., sex, BMI, depressive symptoms). ${ }^{25}$

Little attention has been given to the possibility that the various components of an exercise regimen (e.g., dose, mode, timing) have differential effects on sleep. Experimental studies have typically employed moderate-intensity aerobic exercise or moderate-intensity resistance exercise at doses that approximate public health guidelines; however, direct comparisons between different exercise intensities have not been performed, and combining or directly comparing modes of exercise have been rare. ${ }^{20,26}$ There has also been minimal inquiry into the possible dose-response effects of exercise on sleep ${ }^{27,28}$-for instance, is there a minimal dose of exercise (integrating frequency, duration, and intensity) below which sleep is not improved? Conversely, are greater improvements in sleep observed with higher doses of exercise? Likewise, the time course of change in sleep with exercise is relatively unknown, as most studies have only examined sleep at baseline and postintervention. Whether the timing of exercise matters in relation to sleep has received the 
most attention thus far, primarily due to the alleged sleep-disrupting effect of late-night exercise. While the optimal time of exercise for sleep remains equivocal, ${ }^{13,18}$ experimental evidence does not support the claim that late-night exercise disrupts subsequent sleep. ${ }^{29}$

The efficacy of exercise relative to standard sleep treatment options (e.g., hypnotics, CBT-I, CPAP therapy) is unknown as exercise has rarely been directly compared to these treatments. ${ }^{16}$ Accordingly, comparative-effectiveness trials could establish the true value of exercise to sleep medicine. Similarly, research examining the value of exercise as an adjunct to standard sleep treatment is needed. In particular, while exercise is unlikely to be a viable standalone OSA therapy, it may hold particular promise as an adjunct therapy due to its robust benefits on daytime functioning and cardiovascular risk, two prominent consequences of OSA. ${ }^{30}$

\section{Does Poor Sleep Contribute to Physical Inactivity?}

A bidirectional relationship between exercise and sleep has long been assumed. ${ }^{11}$ Until recently, though, evidence to support the claim that poor sleep led to less exercise was limited to investigations that focused on group differences in physical activity between adults with and without significant sleep disturbances. These studies found, in general, that adults with poor sleep were less active than similar adults without sleep complaints. For instance, adults with insomnia symptoms are less active (e.g., ${ }^{31}$ ) and have lower cardiorespiratory fitness ${ }^{32}$ than adults without insomnia, possibly due to daytime sleepiness and/or fatigue. ${ }^{33}$ Similarly, adults with SDB are less likely to be physically active than adults without SDB, ${ }^{34,35}$ with SDB severity inversely correlated with objective indices of physical activity. ${ }^{36}$ These low activity levels were commonly attributed to the excess weight, low energy, and high levels of fatigue and sleepiness that are characteristics of adults with SDB. ${ }^{37,38}$

Recent studies have attempted to examine the bidirectional relationship between exercise and sleep using more sophisticated statistical approaches. There are only a handful of these studies, and current evidence is limited to samples of older adults or adults with chronic pain. Nevertheless, two studies have shown that poor sleep (i.e., bad sleep quality or insomnia symptoms) predicts lower levels of physical activity 2-7 years later. ${ }^{39,40}$ Even more compelling, though, are studies that have found that nightly variations in various sleep parameters (i.e., sleep quality, ${ }^{41,42}$ subjective sleep latency, ${ }^{24}$ actigraphic sleep efficiency ${ }^{43}$ ) predict physical activity behavior the following day. Some of the observed associations may be of limited practical significance (e.g., a 30-min increase in the time to fall asleep associated with a 1-min decrease in next-day exercise duration, ${ }^{24}$ a $10 \%$ increase in sleep efficiency associated with a $5.4 \%$ increase in next-day MVPA minutes ${ }^{43}$ ). Regardless, it is notable that most of these studies found poor sleep to be a stronger predictor of subsequent physical activity than vice versa. ${ }^{24,39,40,43}$ These findings are also concordant with recent research that has found that experimentally imposed short sleep duration (e.g., $<6$ hours per night) results in lower daytime activity levels. ${ }^{44,45}$

Initial evidence also suggests that one's diurnal preference (i.e., morningness-eveningness) and the timing of sleep-independent of sleep quality and duration — is related to physical 
activity in adults. Specifically, greater morningness and an earlier habitual wake time were associated with greater levels of physical activity. ${ }^{46,47}$ As these two studies were crosssectional analyses, it is possible that exercise (or lack thereof) alters diurnal preference and/or sleep timing. Exercise can shift the timing of circadian rhythms, though whether the timing is advanced or delayed likely depends upon the time of exercise. ${ }^{48}$

\section{Does Improving Sleep Lead to Increased Physical Activity?}

The observational studies noted above allow the possibility that improving sleep may increase daytime activity levels. This is a logical supposition, as sleep and physical activity are both modifiable behaviors, and better sleep-by minimizing daytime impairment (e.g., sleepiness, fatigue/energy)—could facilitate an active lifestyle. However, experimental evidence to support this possibility is lacking. To my knowledge, only one study has examined this question in adults with poor sleep and/or insomnia. In a sample of older adults diagnosed with chronic insomnia, Kline and colleagues found that 4 weeks of behavioral treatment for insomnia did not change either self-reported or actigraphic measures of daytime activity despite significant improvements in subjective and actigraphic sleep at post-intervention. ${ }^{49}$

Similar results have been found in the three studies that have examined whether CPAP treatment increases daytime activity levels in patients with OSA. Across these studies, 1-6 months of CPAP treatment had no discernible impact on objective measures of physical activity despite significant improvement in daytime impairment (e.g., sleepiness, fatigue)..$^{50-52}$ Moreover, a recent study found that an intervention combining CPAP with tailored behavioral modification of eating habits and physical activity successfully improved eating behavior but was unable to change physical activity levels. ${ }^{53}$

The disappointing results of these studies - in particular, those involving OSA patientssuggest that improving sleep is insufficient to spontaneously change physical activity behavior. Traditional sleep treatments may need to be augmented with focused physical activity counseling to change activity levels in adults with poor sleep. Furthermore, identification of the specific barriers and facilitators to altering physical activity behavior in adults with disturbed sleep may be helpful. Although this has not been evaluated in adults with poor sleep quality or insomnia, studies focused on OSA patients have found that physical activity levels correspond to patients' stage of behavior change readiness ${ }^{54}$ and fear of movement. 55

\section{Conclusions}

Both poor/insufficient sleep and physical inactivity are significant public health priorities. Interest in the bidirectional relationship between exercise and sleep has surged in recent years, presumably due to increased recognition of the value of sleep and the modifiability of both sleep and exercise behaviors. However, while intervening on physical activity levels has been shown to improve sleep, improving sleep has not resulted in increased levels of physical activity. Thus, additional research is needed to overcome the current conundrumalthough exercise may be an important behavioral treatment for improving poor and/or 
disordered sleep, poor sleep may be a key impediment to initiating and/or maintaining a physically active lifestyle.

\section{Acknowledgments}

Support provided by K23 HL118318.

\section{References}

1. Luyster FS, Strollo PJ Jr, Zee PC, Walsh JK. Sleep: a health imperative. Sleep. 2012; 35:727-734. [PubMed: 22654183]

2. Terre L. Clinical implications of impaired sleep. Am J Lifestyle Med. In press.

3. Centers for Disease Control and Prevention. Short sleep duration among workers-United States, 2010. MMWR Morb Mortal Wkly Rep. 2012; 61:281-285. [PubMed: 22534760]

4. Institute of Medicine. Sleep disorders and sleep deprivation: an unmet public health problem. Washington, D.C.: National Academies Press; 2006.

5. Roth T, Coulouvrat C, Hajak G, et al. Prevalence and perceived health associated with insomnia based on DSM-IV-TR; International Statistical Classification of Diseases and Related Health Problems, Tenth Revision; and Research Diagnostic Criteria/International Classification of Sleep Disorders, Second Edition criteria: results from the America Insomnia Survey. Biol Psychiatry. 2011; 69:592-600. [PubMed: 21195389]

6. Peppard PE, Young T, Barnet JH, Palta M, Hagen EW, Hla KM. Increased prevalence of sleepdisordered breathing in adults. Am J Epidemiol. 2013; 177:1006-1014. [PubMed: 23589584]

7. Glass J, Lanctot KL, Herrmann N, Sproule BA, Busto UE. Sedative hypnotics in older people with insomnia: meta-analysis of risks and benefits. BMJ. 2005; 331:1169. [PubMed: 16284208]

8. Espie CA. "Stepped care": a health technology solution for delivering cognitive behavioral therapy as a first line insomnia treatment. Sleep. 2009; 32:1549-1558. [PubMed: 20041590]

9. Sawyer AM, Gooneratne NS, Marcus CL, Ofer D, Richards KC, Weaver TE. A systematic review of CPAP adherence across age groups: clinical and empiric insights for developing CPAP adherence interventions. Sleep Med Rev. 2011; 15:343-356. [PubMed: 21652236]

10. Buman MP, King AC. Exercise as a treatment to enhance sleep. Am J Lifestyle Med. 2010; 4:500514.

11. Youngstedt SD, Kline CE. Epidemiology of exercise and sleep. Sleep Biol Rhythms. 2006; 4:215221. [PubMed: 25374476]

12. Yang PY, Ho KH, Chen HC, Chien MY. Exercise training improves sleep quality in middleaged and older adults with sleep problems: a systematic review. J Physiother. 2012; 58:157-163. [PubMed: 22884182]

13. Tworoger SS, Yasui Y, Vitiello MV, et al. Effects of a yearlong moderate-intensity exercise and a stretching intervention on sleep quality in postmenopausal women. Sleep. $2003 ; 26: 830-836$. [PubMed: 14655916]

14. King AC, Pruitt LA, Woo S, et al. Effects of moderate-intensity exercise on polysomnographic and subjective sleep quality in older adults with mild to moderate sleep complaints. J Gerontol A Biol Sci Med Sci. 2008; 63:997-1004. [PubMed: 18840807]

15. Alessi CA, Schnelle JF, MacRae PG, et al. Does physical activity improve sleep in impaired nursing home residents? J Am Geriatr Soc. 1995; 43:1098-1102. [PubMed: 7560698]

16. Guilleminault C, Clerk A, Black J, Labanowski M, Pelayo R, Claman D. Nondrug treatment trials in psychophysiologic insomnia. Arch Intern Med. 1995; 155:838-844. [PubMed: 7717792]

17. Reid KJ, Baron KG, Lu B, Naylor E, Wolfe L, Zee PC. Aerobic exercise improves selfreported sleep and quality of life in older adults with insomnia. Sleep Med. 2010; 11:934-940. [PubMed: 20813580]

18. Passos GS, Poyares D, Santana MG, et al. Effects of moderate aerobic exercise training on chronic primary insomnia. Sleep Med. 2011; 12:1018-1027. [PubMed: 22019457] 
19. Iftikhar IH, Kline CE, Youngstedt SD. Effects of exercise training on sleep apnea: a meta-analysis. Lung. 2014; 192:175-184. [PubMed: 24077936]

20. Kline CE, Crowley EP, Ewing GB, et al. The effect of exercise training on obstructive sleep apnea and sleep quality: a randomized controlled trial. Sleep. 2011; 34:1631-1640. [PubMed: 22131599]

21. Kline CE, Ewing GB, Burch JB, et al. Exercise training improves selected aspects of daytime functioning in adults with obstructive sleep apnea. J Clin Sleep Med. 2012; 8:357-365. [PubMed: 22893765]

22. Youngstedt SD, O'Connor PJ, Dishman RK. The effects of acute exercise on sleep: a quantitative synthesis. Sleep. 1997; 20:203-214. [PubMed: 9178916]

23. Passos GS, Poyares D, Santana MG, Garbuio SA, Tufik S, Mello MT. Effect of acute physical exercise on patients with chronic primary insomnia. J Clin Sleep Med. 2010; 6:270-275. [PubMed: 20572421]

24. Baron KG, Reid KJ, Zee PC. Exercise to improve sleep in insomnia: exploration of the bidirectional effects. J Clin Sleep Med. 2013; 9:819-824. [PubMed: 23946713]

25. Buman MP, Hekler EB, Bliwise DL, King AC. Moderators and mediators of exerciseinduced objective sleep improvements in midlife and older adults with sleep complaints. Health Psychol. 2011; 30:579-587. [PubMed: 21688915]

26. Richards KC, Lambert C, Beck CK, et al. Strength training, walking, and social activity improve sleep in nursing home and assisted living residents: randomized controlled trial. J Am Geriatr Soc. 2011; 59:214-223. [PubMed: 21314643]

27. Kline CE, Sui X, Hall MH, et al. Dose-response effects of exercise training on the subjective sleep quality of postmenopausal women: exploratory analyses of a randomised controlled trial. BMJ Open. 2012; 2:e001044.

28. Singh NA, Stavrinos TM, Scarbek Y, Galambos G, Liber C, Fiatarone Singh MA. A randomized controlled trial of high versus low intensity weight training versus general practitioner care for clinical depression in older adults. J Gerontol A Biol Sci Med Sci. 2005; 60:768-776. [PubMed: 15983181]

29. Brand S, Kalak N, Gerber M, Kirov R, Puhse U, Holsboer-Trachsler E. High self-perceived exercise exertion before bedtime is associated with greater objectively assessed sleep efficiency. Sleep Med. In press.

30. Ackel-D'Elia C, da Silva AC, Silva RS, et al. Effects of exercise training associated with continuous positive airway pressure treatment in patients with obstructive sleep apnea syndrome. Sleep Breath. 2012; 16:723-735. [PubMed: 21805226]

31. Sherrill DL, Kotchou K, Quan SF. Association of physical activity and human sleep disorders. Arch Intern Med. 1998; 158:1894-1898. [PubMed: 9759685]

32. Strand LB, Laugsand LE, Wisloff U, Nes BM, Vatten L, Janszky I. Insomnia symptoms and cardiorespiratory fitness in healthy individuals: the Nord-Trondelag Health Study (HUNT). Sleep. 2013; 36:99-108C. [PubMed: 23288976]

33. Chasens ER, Sereika SM, Weaver TE, Umlauf MG. Daytime sleepiness, exercise, and physical function in older adults. J Sleep Res. 2007; 16:60-65. [PubMed: 17309764]

34. Chasens ER, Sereika SM, Houze MP, Strollo PJ. Subjective and objective appraisal of activity in adults with obstructive sleep apnea. J Aging Res. 2011; 2011:751819. [PubMed: 21253491]

35. Kline CE, Irish LA, Buysse DJ, et al. Sleep hygiene behaviors among midlife women with insomnia or sleep-disordered breathing: the SWAN Sleep Study. J Womens Health. In press.

36. Verwimp J, Ameye L, Bruyneel M. Correlation between sleep parameters, physical activity and quality of life in somnolent moderate to severe obstructive sleep apnea adult patients. Sleep Breath. 2013; 17:1039-1046. [PubMed: 23354507]

37. Hong S, Dimsdale JE. Physical activity and perception of energy and fatigue in obstructive sleep apnea. Med Sci Sports Exerc. 2003; 35:1088-1092. [PubMed: 12840627]

38. Young T, Peppard PE, Taheri S. Excess weight and sleep-disordered breathing. J Appl Physiol. 2005; 99:1592-1599. [PubMed: 16160020]

39. Holfeld B, Ruthig JC. A longitudinal examination of sleep quality and physical activity in older adults. J Appl Gerontol. In press. 
40. Haario P, Rahkonen O, Laaksonen M, Lahelma E, Lallukka T. Bidirectional associations between insomnia symptoms and unhealthy behaviours. J Sleep Res. 2013; 22:89-95. [PubMed: 22978579]

41. Dzierzewski JM, Buman MP, Giacobbi PR Jr, et al. Exercise and sleep in communitydwelling older adults: evidence for a reciprocal relationship. J Sleep Res. 2014; 23:61-68. [PubMed: 23980920]

42. Tang NK, Sanborn AN. Better quality sleep promotes daytime physical activity in patients with chronic pain? A multilevel analysis of the within-person relationship. PLoS One. 2014; 9:e92158. [PubMed: 24667276]

43. Lambiase MJ, Gabriel KP, Kuller LH, Matthews KA. Temporal relationships between physical activity and sleep in older women. Med Sci Sports Exerc. 2013; 45:2362-2368. [PubMed: 23739529]

44. Schmid SM, Hallschmid M, Jauch-Chara K, et al. Short-term sleep loss decreases physical activity under free-living conditions but does not increase food intake under time-deprived laboratory conditions in healthy men. Am J Clin Nutr. 2009; 90:1476-1482. [PubMed: 19846546]

45. Bromley LE, Booth JN III, Kilkus JM, Imperial JG, Penev PD. Sleep restriction decreases the physical activity of adults at risk for type 2 diabetes. Sleep. 2012; 35:977-984. [PubMed: 22754044]

46. Evans DS, Snitker S, Wu SH, et al. Habitual sleep/wake patterns in the Old Order Amish: heritability and association with non-genetic factors. Sleep. 2011; 34:661-669. [PubMed: 21532960]

47. Haraszti RA, Purebl G, Salavecz G, Poole L, Dockray S, Steptoe A. Morningnesseveningness interferes with perceived health, physical activity, diet and stress levels in working women: a cross-sectional study. Chronobiol Int. In press.

48. Atkinson G, Edwards B, Reilly T, Waterhouse J. Exercise as a synchroniser of human circadian rhythms: an update and discussion of the methodological problems. Eur J Appl Physiol. 2007; 99:331-341. [PubMed: 17165050]

49. Kline CE, Lubinski LC, Seres RJ, Miewald JM, Hall MH, Buysse DJ. Improved sleep quality does not result in increased daytime activity in older adults with insomnia [abstract]. Med Sci Sports Exerc. 2014; 46:S434.

50. West SD, Kohler M, Nicoll DJ, Stradling JR. The effect of continuous positive airway pressure treatment on physical activity in patients with obstructive sleep apnoea: a randomised controlled trial. Sleep Med. 2009; 10:1056-1058. [PubMed: 19427263]

51. Diamanti C, Manali E, Ginieri-Coccossis M, et al. Depression, physical activity, energy consumption, and quality of life in OSA patients before and after CPAP treatment. Sleep Breath. 2013; 17:1159-1168. [PubMed: 23386373]

52. Chasens ER, Korytkowski M, Sereika SM, Burke LE, Drumheller OJ, Strollo PJ Jr. Improving activity in adults with diabetes and coexisting obstructive sleep apnea. West J Nurs Res. 2014; 36:294-311. [PubMed: 23976778]

53. Igelstrom, Emtner M, Lindberg E, Asenlof P. Tailored behavioral medicine intervention for enhanced physical activity and healthy eating in patients with obstructive sleep apnea syndrome and overweight. Sleep Breath. In press.

54. Smith SS, Doyle G, Pascoe T, Douglas JA, Jorgensen G. Intention to exercise in patients with obstructive sleep apnea. J Clin Sleep Med. 2007; 3:689-694. [PubMed: 18198801]

55. Igelstrom H, Emtner M, Lindberg E, Asenlof P. Physical activity and sedentary time in persons with obstructive sleep apnea and overweight enrolled in a randomized controlled trial for enhanced physical activity and healthy eating. Sleep Breath. 2013; 17:1257-1266. [PubMed: 23536259] 